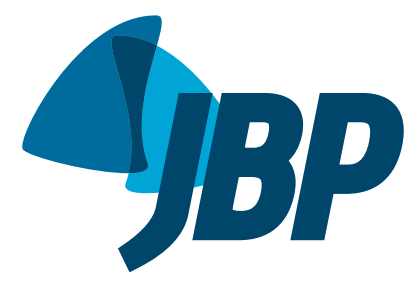

\title{
Control measures to trace $\leq 15$-year-old contacts of index cases of active pulmonary tuberculosis
}

\author{
Cláudia Di Lorenzo Oliveira', Angelita Cristine de Melo², \\ Lílian Ruth Silva de Oliveira ${ }^{3}$, Emerson Lopes Froede ${ }^{1}$, Paulo Camargos ${ }^{3}$
}

1. Curso de Medicina, Universidade Federal de São João del-Rei, Divinópolis, Brasil.

2. Curso de Farmácia, Universidade Federal de São João del-Rei, Divinópolis, Brasil.

3. Programa de Pós-Graduação em Ciências da Saúde, Universidade Federal de São João del-Rei, Divinópolis, Brasil.

Submitted: 7 April 2014

Accepted: 31 May 2015

Study carried out under the auspices of the Programa de Pós-Graduação em Ciências

\begin{abstract}
This was descriptive study carried out in a medium-sized Brazilian city. In $\leq 15$-year-old contacts of index cases of active pulmonary tuberculosis, we assessed compliance with the Brazilian national guidelines for tuberculosis control. We interviewed 43 contacts and their legal guardians. Approximately $80 \%$ of the contacts were not assessed by the municipal public health care system, and only $21 \%$ underwent tuberculin skin testing. The results obtained with the Chi-square Automatic Interaction Detector method suggest that health care teams have a biased attitude toward assessing such contacts and underscore the need for training health professionals regarding tuberculosis control programs.
\end{abstract}

Keywords: Tuberculosis, pulmonarylepidemiology, Tuberculosis, pulmonarylprevention and control; Contact tracing. da Saúde, Universidade Federal de São João Del-Rei, Divinópolis (MG) Brasil.

Assessment of contacts is one of the essential steps in tuberculosis control programs, and its purpose is to diagnose or rule out latent infection or active tuberculosis in such individuals. ${ }^{(1)}$

In Brazil, activities that health care facilities should employ to assess contacts are described in the Brazilian National Ministry of Health guidelines for tuberculosis control. ${ }^{(1)}$ In those guidelines, this assessment involves five steps, namely invitation for contacts to come to the health care facility for assessment, interview by the health care team, tuberculin skin testing, chest X-ray, and, when necessary, prescription of treatment for latent tuberculosis infection (LTBI) or, if appropriate, for active tuberculosis. ${ }^{(1)}$

Despite the strategic importance of such measures, compliance rates are low (up to approximately 60\%) in Brazil, $(2,3)$ whereas, in developed countries, the aforementioned surveillance and control measures reach approximately $90 \%$ of individuals. ${ }^{(4)}$

The objective of the present study was to assess compliance with the steps applicable to $\leq 15$-year-old contacts of index cases of active pulmonary tuberculosis.

This was a descriptive study carried out in a medium-sized city in the central-western portion of the Brazilian state of Minas Gerais. Minas Gerais is the second most populous Brazilian state, as well as being the third most important economically; the city under study serves as a regional hub for health care for 55 cities and had, between 2007 and 2010, an average resident population of 213,501 . $^{(5)}$

The inclusion criteria for contacts were as follows: being $\leq 15$ years of age; living in the city under study; and being a contact of a case of active tuberculosis reported between January of 2007 and December of 2010, according to the Brazilian Case Registry Database. The index cases were located by means of the addresses available on the tuberculosis reporting and investigation forms that feed the database.

Data on contacts were collected in December of 2010 by administering a structured coding instrument, at a home visit; by determining, through a review of the municipal health information system, over a period of up to two years after the index cases had been reported, whether or not tuberculin skin testing and chest X-ray were performed; and by assessing prescription of and adherence to treatment for LTBI, through a review of dispensing records for isoniazid.

The statistical analysis included descriptive tests and bivariate and multivariate analyses in which the response variable was compliance or noncompliance with the steps in the assessment of contacts, as determined by the Chi-square Automatic Interaction Detector (CHAID) algorithm, ${ }^{(6)}$ with cross-validation of results of 10 subsamples, a maximum of 10 nodes, and 5 cases in the child node. Pearson's chi-square test with Bonferroni's adjustment was used for between-node separation. Explanatory variables

\section{Correspondence to:}

Paulo Camargos.

Rua do Ouro, 1200/502, CEP 30220-000, Belo Horizonte, MG, Brasil.

Tel.: 5531 3267-4879 or 5531 9976-4879. Fax: 5531 3409-9664.

E-mail: paulo.camargos@pq.cnpq.br Financial support: This study received partial funding from the Fundação de Amparo à Pesquisa do Estado de Minas Gerais (FAPEMIG

Foundation for the Support of Research in the state of Minas Gerais; Process no. CDS/APQ/00817/12). Paulo Camargos is the recipient of research productivity grants from the Brazilian Conselho Nacional de Desenvolvimento Cientifico e Tecnológico (CNPq, National Council for Scientific and Technological Development) and from

FAPEMIG (Process no. PPM 00065/14), under the "Programa Pesquisador Mineiro". 
were degree of contact and family relationship between contacts and the index case, gender, number of rooms in the index case's house, and number of residents in the household. For the analysis of the second step (interview by the health care team), we included, in addition to the aforementioned variables, home visits (yes/no) and invitation for contacts to come to the health care facility.

The research project and its written informed consent form were submitted to and approved by the Human Research Ethics Committee of the Hospital São João de Deus - the only such committee in the city at the time the study was conducted-Protocol no. 63/2011.

Between January of 2007 and December of 2010, 135 cases of tuberculosis were reported, of which 54

(A) Step 1

Invitation from any type of health care facility

\begin{tabular}{|lcc|}
\hline Cat & $\%$ & $\mathrm{n}$ \\
\hline Yes & 55.81 & 24 \\
No & 44.19 & 19 \\
\hline Total & $(100.00)$ & 43 \\
\hline
\end{tabular}

Number of rooms in the residence

$\mathrm{P}$-value $=0.0006 ;$ Chi-square $=11.8824 ; \mathrm{df}=1$

\begin{tabular}{|lll|}
\hline \multicolumn{1}{|c|}{\begin{tabular}{|c|} 
Up to six rooms \\
Cat
\end{tabular}} & $\%$ & $\mathrm{n}$ \\
\hline Yes & 76.92 & 20 \\
No & 23.08 & 6 \\
\hline Total & $(60.47)$ & 26 \\
\hline
\end{tabular}

(C) Step 3

PPD testing performed

\begin{tabular}{|ccc|}
\hline Cat & $\%$ & $\mathrm{n}$ \\
\hline Yes & 20.93 & 9 \\
No & 79.07 & 34 \\
\hline Total & $(100.00)$ & 43 \\
\hline
\end{tabular}

Contact was physician assessed

$\mathrm{p}$-value $=0.0000 ;$ Chi-square $=22.2265 ; \mathrm{df}=1$

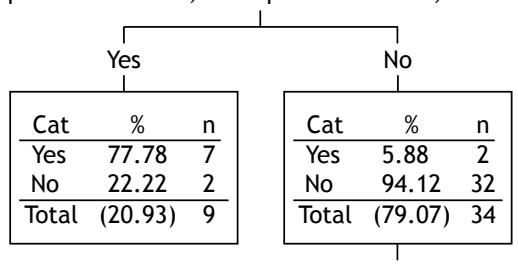

Interview by the health care team $\mathrm{p}$-value $=0.0151 ;$ Chi-square $=5.9028 ; \mathrm{df}=1$

\begin{tabular}{|ccc|}
\hline Yes \\
Cat & $\%$ & $\mathrm{n}$ \\
\hline Yes & 22.22 & 2 \\
No & 77.78 & 7 \\
\hline Total & $(20.93)$ & 9 \\
\hline Yes & 0.00 & 0 \\
\hline To & 100.00 & 25 \\
\hline Total & $(58.14)$ & 25 \\
\hline
\end{tabular}

( $40 \%$ ) consisted of patients with active pulmonary tuberculosis and who therefore were eligible for the study. We excluded 11 patients (20.4\%) because there was no record of their addresses, 18 (34.2\%) because they did not meet the inclusion criteria-13 (24.0\%) because they had no $\leq 15$-year-old contacts at the time of diagnosis and $5(9.2 \%)$ because they lived in other cities-and $2(3.7 \%)$ because they declined to participate in the study. There thus remained 21 index cases $(38.9 \%)$, who were revisited between July of 2011 and February of 2013 and through whom 43 contacts who met the inclusion criteria were identified.

Table 1 presents the general characteristics of the study population.

(B) Step 2

Interview by the health care team

\begin{tabular}{|lcc|}
\hline Cat & $\%$ & $\mathrm{n}$ \\
\hline Yes & 34.88 & 15 \\
No & 65.12 & 28 \\
\hline Total & $(100.00)$ & 43 \\
\hline
\end{tabular}

Invitation from any type of health care facility P-value $=0.0000 ;$ Chi-square $=18.2366 ; \mathrm{df}=1$

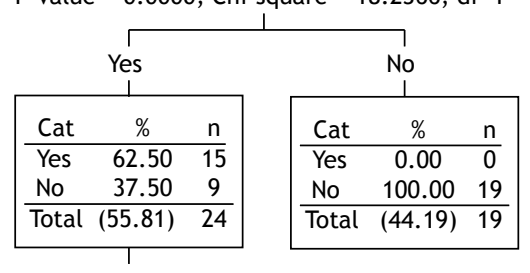

Duration of contact

$\mathrm{P}$-value $=0.0054 ;$ Chi-square $=7.7257 ; \mathrm{df}=1$

\begin{tabular}{|lcc|}
\multicolumn{1}{c}{ Up to 8 weeks } & \multicolumn{3}{c|}{ More than 8 weeks } \\
\hline Cat & $\%$ & $\mathrm{n}$ \\
\hline Yes & 85.71 & 12 \\
No & 14.29 & 2 \\
\hline Total & $(32.56)$ & 14 \\
\hline
\end{tabular}$\quad$\begin{tabular}{|ccc} 
Cat & $\%$ & $\mathrm{n}$ \\
\hline Yes & 30.00 & 3 \\
No & 70.00 & 7 \\
\hline Total & $(23.26)$ & 10 \\
\hline
\end{tabular}


Table 1. Descriptive characteristics of $\leq 15$-year-old contacts of index cases of active pulmonary tuberculosis. ${ }^{a}$

\begin{tabular}{|c|c|c|c|c|}
\hline \multirow[t]{2}{*}{ Characteristic } & \multirow[t]{2}{*}{$N=43$} & \multicolumn{2}{|c|}{$\begin{array}{c}\text { Invitation from the PHC team to be } \\
\text { assessed for health status }\end{array}$} & \multirow[t]{2}{*}{ p* } \\
\hline & & $\begin{array}{l}\text { Invited } \\
(\mathrm{n}=24)\end{array}$ & $\begin{array}{l}\text { Not invited } \\
\text { (n }=19)\end{array}$ & \\
\hline \multicolumn{5}{|l|}{ Sociodemographic } \\
\hline Male gender & $22(51.2)$ & $12(50.0)$ & $10(52.6)$ & $>0.05$ \\
\hline Age at interview ${ }^{b}$ & $10.2(8.3-12.5)$ & $10.5(8.6-12.7)$ & $10.0(7.6-12.2)$ & $>0.05$ \\
\hline Knowing how to read or write & $35(81.4)$ & $20(83.3)$ & $15(78.9)$ & $>0.05$ \\
\hline \multicolumn{5}{|l|}{ Residence type } \\
\hline Owned & $32(74.4)$ & $17(70.8)$ & $15(78.9)$ & \\
\hline Rented & $10(23.3)$ & $7(29.2)$ & $3(15.8)$ & \\
\hline Borrowed & $1(2.3)$ & - & $1(5.3)$ & \\
\hline Number of rooms & & & & 0.01 \\
\hline Up to four & $3(7.0)$ & $2(8.3)$ & $1(5.3)$ & \\
\hline Five & $13(30.2)$ & 9 (37.5) & $4(21.1)$ & \\
\hline Six & $10(23.3)$ & $9(37.5)$ & $1(5.3)$ & \\
\hline Seven or more & $17(39.5)$ & $4(16.7)$ & $13(68.4)$ & \\
\hline \multicolumn{2}{|l|}{ Number of residents in the household } & & & $<0.01$ \\
\hline Up to four & $14(32.6)$ & $11(45.8)$ & $3(15.8)$ & \\
\hline Five or more & $28(65.1)$ & $12(40.0)$ & $16(85.2)$ & \\
\hline No response & $1(2.3)$ & $1(4.2)$ & - & \\
\hline \multicolumn{5}{|l|}{ Sanitation status } \\
\hline Piped, treated water & $43(100.0)$ & $24(100.0)$ & $19(100.0)$ & $>0.05$ \\
\hline Sewage collection system & $38(88.4)$ & $20(83.3)$ & $18(94.7)$ & $>0.05$ \\
\hline Garbage collection & $43(100.0)$ & $24(100.0)$ & $19(100.0)$ & $>0.05$ \\
\hline \multicolumn{5}{|l|}{ Contact with the index case } \\
\hline \multicolumn{5}{|l|}{ Frequency of contact } \\
\hline Daily & $25(58.1)$ & $15(62.5)$ & $10(52.6)$ & $>0.05$ \\
\hline Residence & $20(80.0)$ & $13(86.7)$ & $7(70.0)$ & \\
\hline Same lot & $5(20.0)$ & $2(13.3)$ & $3(30.0)$ & \\
\hline Infrequent & $18(41.9)$ & 9 (37.5) & $9(47.4)$ & \\
\hline Duration of contact & & & & $<0.01$ \\
\hline$\leq 2$ weeks & $12(27.9)$ & $9(37.5)$ & $3(15.8)$ & \\
\hline 3-10 weeks & $14(32.6)$ & $9(37.5)$ & $5(26.3)$ & \\
\hline$>10$ weeks & $17(39.5)$ & $5(24.0)$ & $11(57.9)$ & \\
\hline \multicolumn{3}{|c|}{ Degree of family relationship with the index case } & & 0.03 \\
\hline Grandchild & $16(37.2)$ & $11(45.8)$ & $5(26.3)$ & \\
\hline Child & $12(27.9)$ & $8(33.8)$ & $4(21.1)$ & \\
\hline Nephew/niece & $12(27.9)$ & $2(8.3)$ & $10(52.6)$ & \\
\hline Other & $2(4.7)$ & $2(8.3)$ & - & \\
\hline Sibling & $1(2.3)$ & $1(4.2)$ & - & \\
\hline $\mathrm{PHC}$ received by contacts & & & & $>0.05$ \\
\hline \multicolumn{5}{|l|}{ Type of health care facility } \\
\hline Family health care clinic & $8(18.6)$ & $5(20.8)$ & $3(15.8)$ & \\
\hline Primary health care clinic & $35(81.4)$ & $19(79.2)$ & $16(84.2)$ & \\
\hline \multicolumn{5}{|l|}{ Preventive activities } \\
\hline BCG vaccination & & & & $>0.05$ \\
\hline First vaccination & $42(97.7)$ & $23(95.8)$ & $19(100.0)$ & \\
\hline Revaccination & $1(2.3)$ & $1(4.2)$ & - & \\
\hline $\begin{array}{l}\text { Invitation from the PHC team to } \\
\text { be assessed for health status }\end{array}$ & $24(55.8)$ & $24(100.0)$ & - & - \\
\hline Invitation accepted & $15(62.5)$ & $15(62.5)$ & - & $<0.01$ \\
\hline Home visit & $7(43.8)$ & $7(46.7)$ & - & 0.01 \\
\hline $\begin{array}{l}\text { Assessment by the } \\
\text { physician }^{c}\end{array}$ & $9(56.2)$ & $8(53.3)$ & $1(100.0)$ & 0.03 \\
\hline Underwent tuberculin skin testing & $9(20.9)$ & $8(33.3)$ & $1(5.3)$ & 0.03 \\
\hline Underwent chest X-ray & $5(11.6)$ & $4(16.7)$ & $1(5.3)$ & $>0.05$ \\
\hline Underwent chemoprophylaxis & $1(14.3)$ & $1(4.2)$ & - & $>0.05$ \\
\hline
\end{tabular}

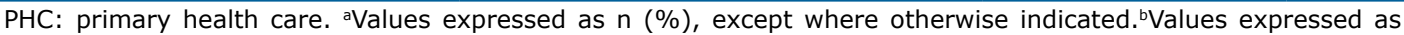
median (interquartile range). 'One contact who was not invited for assessment sought the physician by itself. Therefore, the total number of assessments performed by the PHC team was 16 rather than 15. *Pearson's chisquare test with Bonferroni's adjustment. 
Of the 43 contacts, all had received BCG vaccination, $22(51.2 \%)$ were male, $40(93.0 \%)$ lived with five or more persons in the household, $25(58.1 \%)$ had frequent contact with the index case, and 25 (58.1\%) reported a family income of up to two times the national minimum wage.

Figure 1 contains the decision tree for the first three steps in the assessment of contacts. In it, the lack of invitation for contacts to come to the health care facility (in $44.2 \%$; Figure $1 \mathrm{~A}$ ) is of note, as are the lack of interview by the health care team (in $65.1 \%$; Figure $1 B$ ) and the lack of tuberculin skin testing (in $79.1 \%$; Figure $1 \mathrm{C}$ ). In addition, invitation for contacts to come to the health care facility was found to be associated only with number of rooms in the house (a proxy for socioeconomic status), the invitation being made mostly to those living in houses with fewer rooms (76.9\%; Figure $1 \mathrm{~A}$ ). No statistical significance was found for either degree of contact or type of health care facility where care was provided.

Whether an interview would be conducted with contacts and their families was determined by contacts having been instructed to come to the health care facility and by duration of contact. Only 24 contacts (55.8\%; Figure $1 \mathrm{~A}$ ) were invited to come to the health care facility, whereas, among these, the attendance rate was $62.5 \%$.

The most significant variable $(p<0.0001)$ in determining whether a contact would undergo tuberculin skin testing was medical assessment, i.e., $77.8 \%$ of those who were assessed by a physician underwent such testing, which denotes that assessment of contacts was centered on only one professional. In cases in which the contact was not assessed by a physician, the second explanatory variable was interview by the health care team (Figure 1B).

The last two steps in the assessment of contacts were not included in the decision tree because they were not performed for all contacts; they are recommended based on the results obtained in the previous assessment step, i.e., whether a contact will undergo chest X-ray is dependent on tuberculin skin testing results, and whether a contact will receive treatment for LTBI or active tuberculosis is dependent both on chest X-ray findings and tuberculin skin testing results.

Treatment for LTBI should have been recommended to at least 2 of the contacts; however, only 1 of them $(2.3 \%)$ received such treatment and for only about 30 days.

Our review of the literature found no studies on assessment of contacts in which the CHAID method was used, this method being a strategy for statistical analysis that allows the identification of critical points in each step in the investigation of such individuals.

As shown in the present study, assessment of contacts revealed that compliance rates were lower than desirable. A study conducted in the state of Mato Grosso, Brazil, reported that $60.5 \%$ of the contacts younger than 15 years were investigated, and that the proportion of contacts investigated was $40.0 \%$ higher among those exposed to active cases, being higher than that found in the present study. ${ }^{(3)}$ In the city of São José do Rio Preto, Brazil, $63.1 \%$ of the contacts were assessed by the municipal health care system in 2002. (2) Nearly the reverse of this situation is found in developed countries; there, the proportion of contacts who are investigated ranges from $80 \%$ to $90 \%$, as is the case in the USA and in the Netherlands. $(4,7)$

It is possible that biases occurred in the approach to contacts. One such bias is that only the low socioeconomic conditions of the contacts (perceived perhaps because of the size of the houses, given that approximately $60 \%$ had six rooms at most; Table 1 ) would favor the transmission of tuberculosis. However, in the present study, this was not observed, since only $11.6 \%$ of the contacts lived with more than five people in houses with up to six rooms. Another such bias is that the health professionals seem to have assumed that the risk of developing the disease would be higher among those $60 \%$ (Table 1 ) whose duration of exposure to the active source case had occurred within the first 10 weeks than would among those whose duration was greater than 10 weeks $(p<0.01)$. In other words, these professionals believed that, if contacts had not contracted tuberculosis up to that time, they would not develop the disease in the future. This perception is completely wrong. In addition, the present study found that assessment of contacts was physician-centered, contrary to the Brazilian National Tuberculosis Control Program guidelines, which strongly encourage the participation of all health professionals. ${ }^{(2)}$

In conclusion, the present study underscores the urgent need for training primary health care teams on how to approach contacts appropriately, especially those who are exposed to patients with active tuberculosis.

\section{ACKNOWLEDGMENTS}

The authors would like to express their gratitude to Abiqueila da Silva Conceição, Bruna Carolina Lapertosa Santos, Bruna Pereira Bicalho, and Samuel Milanez Carvalho-undergraduate medical students at the Universidade Federal de São João Del-Rei-for their assistance during the home visits and the administration of the questionnaires.

\section{REFERENCES}

1. Brasil. Ministério da Saúde. Secretaria de Vigilância em Saúde. Departamento de Vigilância Epidemiológica. Manual de recomendações para o controle da tuberculose no Brasil. Brasília: Ministério da Saúde; 2011.

2. Gazetta CE, Ruffino-Netto A, Pinto Neto JM, Santos Mde L, Cury

MR, Vendramini SH, et al. Investigation of tuberculosis contacts in the tuberculosis control program of a medium-sized municipality in the southeast of Brazil in 2002. J Bras Pneumol. 2006;32(6):559-65. http://dx.doi.org/10.1590/S1806-37132006000600014

3. Hartwig SV, Ignotti E, Oliveira BF, Pereira HC, Scatena JH. Evaluation 
of surveillance of contacts of new tuberculosis cases in the state of Mato Grosso - Brazil. J Bras Pneumol. 2008:34(5):298-303. http:// dx.doi.org/10.1590/S1806-37132008000500009

4. Anger HA, Proops D, Harris TG, Li J, Kreiswirth BN, Shashkina E, et al. Active case finding and prevention of tuberculosis among a cohort of contacts exposed to infectious tuberculosis cases in New York City. Clin Infect Dis. 2012;54(9):1287-95. http://dx.doi.org/10.1093/ $\mathrm{cid} / \mathrm{cis} 029$

5. Brasil. Ministério da Saúde. Departamento de Informática do SUS DATASUS [homepage on the Internet]. Brasilia: Ministério da Saúde [cited 2015 Feb 12]. População Residente - Minas Gerais. Available from: http:/tabnet.datasus.gov.br/cgi/tabcgi.exe?ibge/cnv/popmg.def
6. Von Zuben FJ, Attux RR. Notas de aula IA004. Tópico 7 - Árvores de Decisão [database on the Internet]. Campinas: Unicamp. Faculdade de Engenharia Elétrica e de Computação. Departamento de Engenharia de Computação e Automação Industrial [cited 2015 Feb 12]. [Adobe Acrobat document, 44p.]. Available from: ftp://ftp. dca.fee.unicamp.br/pub/docs/vonzuben/ia004_1s10/notas_de_aula/ topico7_IA004_1s10.pdf

7. Mulder C, Erkens CG, Kouw PM, Huisman EM, Meijer-Veldman W, Borgdorff MW, et al. Missed opportunities in tuberculosis control in The Netherlands due to prioritization of contact investigations. Eur J Public Health. 2012;22(2):177-82. http://dx.doi.org/10.1093/eurpub/ckr017 